(GM-CSF) could enhance the restoration of cells' normal phenotype. However, previous studies suggested GM-CSF might induce hypercoagulability. In this study, we discussed the effect of GM-CSF on coagulation and fibrinolysis after artery deendothelialization.

Methods 24 male New Zealand White rabbits underwent primary iliac artery deendothelialization were randomised to two groups (GM-CSF group and control group). GM-CSF group animals received a subcutaneous injection of GM-CSF, the control group animals were given a subcutaneous injection of equivalent saline. The iliac arteries of all animals were damaged by balloon after 7 days. The plasma levels of tissue-type plasminogen activator (t-PA), plasminogen activator inhibitor-1 (PAI-1) and nitric oxide (NO) were detected before, 1 week, 2 weeks and 4 weeks after angioplasty. The repair and hyperplasia of intima were observed by microscope.

Results The NO levels of the GM-CSF group were higher than that of the control group 2 weeks and 4 weeks after operation $[(91.9 \pm 11.6) \mu \mathrm{mol} / \mathrm{l} \mathrm{vs}(81.7 \pm 12.2) \mu \mathrm{mol} / \mathrm{l}]$; $[(97.7 \pm 10.1) \mu \mathrm{mol} / \mathrm{l} \mathrm{vs}$ (83.2 \pm 12.6$) \mu \mathrm{mol} / \mathrm{l}]$. Although there was no difference between the two groups in both activities of t-PA and PAI- 1 in each specific time point, in 1, 2, 4 weeks after operation, the activities of t-PA became higher than the ones before operation, while the activities of PAI-1 stayed almost the same. Pathological examination showed that the level of intima hyperplasia. VSMC and fibrous tissue of neointima were much lower in GM-CSF group and endothelium was more integrated and smooth.

Conclusion GM-CSF could facilitate the repair of intima, better the function of endothelium without disturbing the balance of coagulation and fibrinolysis.

\section{e0091 POTENTIAL EFFECT OF GRANULOCYTEMACROPHAGE COLONYSTIMULATING FACTOR ON THE ARTERIAL REMODELLING AT THE REFERENCE SITE AFTER ANGIOPLASTY}

doi:10.1136/hrt.2010.208967.91

Ma Xiaojing, Zhang Xinghua. Provincial Hospital Affiliated To Shandong University

Objective Arterial remodelling may also occur at the reference site. Resent studies showed that granulocyte-macrophage colony-stimulating factor (GM-CSF) could facilitate the repair of endothelium and reduce vascular inflammation. To observe the effect of granulocyte-macrophage colony-stimulating factor on arterial remodelling, we evaluated the remodelling at the reference site and the lesion site in rabbit model.

Methods 28 healthy New Zealand White rabbits were randomised to two groups (GM-CSF group and control group). GM-CSF group received a subcutaneous injection of GM-CSF $(10 \mathrm{ug} / \mathrm{kg} / \mathrm{d})$, the control group were given a subcutaneous injection of equivalent saline. The iliac arteries of all animals were damaged by balloon after $7 \mathrm{~d}$. The levels of nitrogen monoxide (NO) were detected before and 4 weeks after angioplasty. Histological sections of iliac from rabbits killed 4 weeks after angioplasty were analysed. Luminal area (LA), external elastic lamina area (EEL), and intimal plus medial areas $(I+M)$ were measured at the lesion $(\mathrm{L})$ and reference $(\mathrm{R})$ sites.

Results The NO level of the GM-CSF group was significantly higher than that of the control group after 4 weeks $(97.69 \pm 10.21$ vs $83.18 \pm 12.57 \mu \mathrm{mol} / \mathrm{l})$. Morphometric analysis showed that the LA (L) of control group was smaller than that of the GM-CSF group $\left(0.87 \pm 0.40\right.$ vs $\left.1.34 \pm 0.52 \mathrm{~mm}^{2}, \mathrm{p}<0.05\right)$ and $\mathrm{I}+\mathrm{M}(\mathrm{L})$ was greater than that of the GM-CSF group $\left(2.62 \pm 0.48\right.$ vs $2.26 \pm 0.43 \mathrm{~mm}^{2}$, $\mathrm{p}<0.05)$. There was no difference in EEL $(\mathrm{L})$ between the two groups $(3.48 \pm 0.80$ vs $3.60 \pm 0.91, p>0.05)$. Morphometric analysis also showed a smaller LA $(\mathrm{R})$ in the control group $(1.60 \pm 0.48$ vs $\left.1.99 \pm 0.54 \mathrm{~mm}^{2}, \mathrm{p}<0.05\right)$, whereas there was no difference in I+M $(\mathrm{R})$ between the two groups. EEL $(\mathrm{R})$ significantly correlated with LA $(\mathrm{R})$, EEL ( $\mathrm{L})$, and $\mathrm{I}+\mathrm{M}(\mathrm{R})$ in both groups combined $(\mathrm{r}=0.91$, $\mathrm{p}<0.0001 ; \mathrm{r}=0.909, \mathrm{p}<0.0001 ;$ and $\mathrm{r}=0.685 ; \mathrm{p}<0.0001$, respectively). LA ( $\mathrm{R}$ ) also correlated with LA ( $\mathrm{L})(\mathrm{r}=0.919 ; \mathrm{p}<0.0001)$.

Conclusion Remodelling can affect both the lesion and the reference sites and appears to occur in parallel and proportionately at both sites. GM-CSF treatment increased reendothelialization of the injured artery and inhibited unfavourable remodelling.

\section{e0092 AVE0991 IMPROVES LEFT VENTRICULAR REMODELLING AND CARDIAC FUNCTION INDUCED BY MYOCARDIAL INFARCTION IN RATS}

doi:10.1136/hrt.2010.208967.92

Zeng Wutao, Chen Weiyan, Leng Xiuyu, Li Cuiling, Lin Hong, Dai Gang. Division of Cardiology, Cardiovascular Medical Department, The First Affiliated Hospital of Sun Yat-sen University, Guangzhou, China

Objective To evaluate the effects of 4-week treatment with the nonpeptide angiotensin-(1-7) analogue AVE 0991 on heart function and left ventricular remodelling induced by myocardial infarction (MI).

Methods In this study, we evaluated the cardiac effects of AVE 0991 on normal and infracted male Sprague-Dawley (SD) rats. MI was induced by left coronary artery ligation. After 4 weeks of treatment, transthoracic echocardiography (TTE) was used to evaluate cardiac function. The heart wet weight was recorded, normalised for body weight. Left ventricle serial sections were dyed with triphenyltetrazolium chloride (TTC) stain to quantify the infarct size, with Masson's trichrome stain to quantify collagen volume fraction $(\mathrm{CVF})$, and with haematoxylin-eosin (HE) stain for diameter measurement of cardiomyocytes.

Results Infarct size was slightly reduced in AVE 0991 group compared to control group $(42.6 \pm 3.6 \%$ vs $50.9 \pm 4.4 \%, p<0.01)$. In addition, AVE 0991 treatment attenuated the decrease in LVFS $(25.54 \pm 7.33 \%$ vs $18.41 \pm 3.32 \%, \mathrm{p}<0.05)$ and LVEF $(54.82 \pm 11.63 \%$ vs $42.7 \pm 6.5 \%, p<0.05)$ compared to control group. AVE 0991 also reduced MI-induced hypertrophy as quantified by diameter measurements of cardiomyocytes (vs. control group $25.49 \pm 4.37 \mu \mathrm{m}$ vs $32.06 \pm 6.85 \mu \mathrm{m}, \mathrm{p}<0.05)$

Conclusion The nonpeptide angiotensin-(1-7) analogue AVE 0991 has a cardioprotective effect on impairment of heart function and ventricular remodelling induced by MI.

\section{e0093 MYOCARDIAL INFARCTION-INDUCED REMODELLING AND INFLAMMATORY CYTOKINES IN RATS ARE ATTENUATED BY AVE0991}

doi:10.1136/hrt.2010.208967.93

Zeng Wutao, Chen Weiyan, Leng Xiuyu, Li Cuiling, Lin Hong, Dai Gang. Division of Cardiology, Cardiovascular Medical Department, The First Affiliated Hospital of Sun Yat-sen University, Guangzhou, China

Objective To investigate the effects of 4-week treatment with the nonpeptide angiotensin-(1-7) analogue AVE 0991 on expression of inflammatory cytokines and remodelling induced by myocardial infarction (MI).

Methods In this study, MI was induced by left coronary artery ligation in male Sprague-Dawley (SD) rats. After 4 weeks of treatment, the heart wet weight was recorded, normalised for body weight. Left ventricle serial sections were dyed with triphenyltetrazolium chloride (TTC) stain to quantify the infarct size, with Masson's trichrome stain to quantify collagen volume fraction (CVF), and with haematoxylin-eosin (HE) stain for diameter measurement of cardiomyocytes. RT-PCR was used to investigate the synthesis of TGF- $\beta 1$, TNF- $\alpha$, collagen type I and III. 